\section{A wide range of patients with RA benefit from anti-TNF therapy}

Randomized controlled trials (RCTs) are the gold standard for evaluating new treatments; however, RCTs often exclude patients with comorbidities, concomitant medications, particular dosing schemes and disease states, so their settings are artificial. An observational cohort study has now found that patients who fulfill the inclusion criteria for the RCTs that led to approval of tumor necrosis factor (TNF) inhibitors for the treatment of rheumatoid arthritis (RA) reflect only a small proportion of patients treated with TNF inhibitors in practice. The study also showed that patients with RA who would not have been eligible for these RCTs could benefit from anti-TNF therapy.

The study included registry data from 1,458 participants in RABBIT, a German observational cohort study that monitors patients with RA who initiate treatment with any licensed biologic agent. Laboratory and clinical outcomes were assessed at baseline and 6 months. Patients were analyzed according to their eligibility (or lack thereof) for inclusion in the five major RCTs of TNF inhibitors.

In this cohort, only $21-33 \%$ of patients would have been eligible for inclusion in one of the major RCTs, but the absolute response rates to TNF inhibitors were comparable in patients who would and who would not have been eligible for the major RCTs. The authors conclude that RCTs need not be so restrictive in their inclusion criteria.

Original article Zink A et al. (2006) Effectiveness of tumor necrosis factor inhibitors in rheumatoid arthritis in an observational cohort study: comparison of patients according to their eligibility for major randomized trials. Arthritis Rheum 54: 3399-3407

\section{Potential anti-inflammatory role of paracetamol in knee OA}

Two similar pilot studies have shown that paracetamol can reduce synovial effusion and tissue volumes in patients with knee osteoarthritis (OA). Previously, paracetamol had not been considered to have anti-inflammatory properties, although it was as effective as ibuprofen for treating the pain of knee OA. In these pilot studies, the investigators aimed to elucidate the relationship between synovial inflammation and joint pain, in patients with knee OA whose pain was treated with paracetamol.

In the first study, 10 patients with knee OA who were taking NSAIDs for their pain, and who then experienced moderately severe pain after a washout period off NSAIDs, underwent baseline MRI. Immediately after the MRI, the patients resumed their NSAID therapy; those patients who experienced $\mathrm{a} \geq 50 \%$ reduction in pain had a second MRI 14 days after the first. The second study enrolled 20 patients with knee OA in a similar protocol with paracetamol up to $4 \mathrm{~g}$ daily. In both studies, synovial effusion and tissue volumes were calculated from the MRI scans.

Synovial effusion and tissue volumes decreased after resumption of NSAID use by $3.3 \mathrm{ml}$ and $1.9 \mathrm{ml}$, respectively, compared with baseline; similarly, these parameters decreased after resumption of paracetamol use by $4.5 \mathrm{ml}$ and $2.7 \mathrm{ml}$, respectively, compared with baseline.

Although these studies were not controlled, were not dose ranging, and did not compare the same patients, they showed that paracetamol might have an anti-inflammatory effect in knee OA. Further studies to elucidate the anti-inflammatory mechanism of action of paracetamol are required.

Original article Brandt KD et al. (2006) Acetaminophen,

like conventional NSAIDs, may reduce synovitis in osteoarthritic knees. Rheumatology 45: 1389-1394

\section{Biomarkers predict and pre-emptive prednisone therapy averts severe SLE flares}

The use of biomarkers to predict disease flare and to indicate a need for pre-emptive treatment in patients with systemic lupus erythematosus (SLE) remains controversial. Tseng et al. studied 154 patients with quiescent SLE, to determine whether changes in levels of complement C3a and autoantibodies to double-stranded (ds) DNA could predict flares.

Anti-dsDNA antibodies, C3a and other biomarkers were measured at monthly intervals. Patients who experienced a serologic flare (an increase of $25 \%$ in anti-dsDNA antibody titer and an increase of $50 \%$ in C3a levels compared with the previous 1-2 months' measurements) were randomly allocated to receive either prednisone $(n=20)$ or placebo $(n=21)$ and were followed up for 90 days. A severe SLE flare was experienced by significantly 